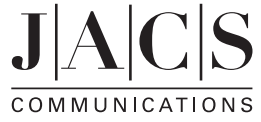

Published on Web 05/10/2006

\title{
The Hidden Role of Acetate in the PbSe Nanocrystal Synthesis
}

\author{
Arjan J. Houtepen, ${ }^{*, \dagger}$ Rolf Koole, ${ }^{*}, \dagger$ Daniël Vanmaekelbergh, ${ }^{\dagger}$ Johannes Meeldijk, ${ }^{\ddagger}$ and

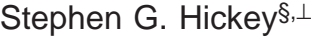 \\ Condensed Matter and Interfaces, University Utrecht, POB 80000, 3508 TA Utrecht, The Netherlands, Electron \\ Microscopy Utrecht, University Utrecht, POB 80.056, 3508 TB Utrecht, The Netherlands, and Institute for Physical \\ Chemistry, University of Hamburg, Grindelallee 117, D-20146 Hamburg, Germany
}

Received March 17, 2006; E-mail: a.j.houtepen@phys.uu.nl; r.koole@phys.uu.nl

In recent years interest in lead chalcogenide colloidal nanocrystals (NCs) has grown considerably. Their rock-salt lattice leads to an electronic band structure different from that of the typical II-VI compounds with important consequences for the optical and electrical properties in the regime of strong confinement. Furthermore, lead chalcogenide quantum dots hold promise for a wide number of opto-electrical applications in the near infrared. ${ }^{1-3}$ Murray reported the first hot injection synthesis of monodisperse PbSe quantum dots. ${ }^{2}$ In subsequent work, a myriad of geometries of PbSe NCs were reported. ${ }^{4-7}$ Surprisingly, the dominant growth mechanism of these geometries appeared to be the oriented attachment of small PbSe NCs. ${ }^{4}$

We observed that it is crucial to use lead precursors completely free of acetate in order to obtain spherical PbSe NCs. In this communication, we present a systematic study of the role of acetate in the growth mechanism and the resulting geometry of the NCs. We show that the presence of even trace amounts of acetate in the reaction mixture leads to starlike geometries formed by oriented attachment. We propose that the presence of acetate is responsible for many of the crystal shapes reported in the literature. In addition, we present the first example of 2-D ordering and crystal alignment of monodisperse, starlike NCs.

The preparation of the $\mathrm{Pb}$-oleate precursor, used for the hot injection synthesis, ${ }^{2}$ involves the following chemical reaction:

$$
\begin{aligned}
\mathrm{PbAc}_{2} \cdot 3 \mathrm{H}_{2} \mathrm{O}+2 \mathrm{C}_{17} \mathrm{H}_{33} \mathrm{COOH} \rightleftarrows \\
\quad \mathrm{Pb}\left(\mathrm{C}_{17} \mathrm{H}_{33} \mathrm{COO}\right)_{2}+3 \mathrm{H}_{2} \mathrm{O}+2 \mathrm{HAc}
\end{aligned}
$$

Hence, if the $\mathrm{Pb}$-oleate precursor is not completely dried, a significant amount of water and acetate will be present in the reaction mixture. Our first attempts to synthesize $\mathrm{PbSe}$ NCs resulted in what appeared to be "stars of David". The $\mathrm{Pb}$-oleate was prepared by heating the precursors to $120{ }^{\circ} \mathrm{C}$ for $30 \mathrm{~min}$, possibly insufficient for complete drying (see Supporting Information).

To obtain spherical NCs, we found that it is crucial to completely dry the $\mathrm{Pb}$-oleate precursor mixture by heating to $>70{ }^{\circ} \mathrm{C}$ under vacuum $\left(<10^{-3}\right.$ mbar) for more than $1 \mathrm{~h}$; see Figure 1A. Therefore we performed a systematic investigation on the effect of water and acetate on the shape and size of PbSe NCs. This was done by completely drying the precursor mixture and adding controlled amounts of water and acetic acid.

The addition of a stoichiometric amount of water or an even larger amount $\left(\mathrm{Pb}: \mathrm{H}_{2} \mathrm{O}=1: 15\right)$ to the carefully dried injection mixture did not result in a deviation from the spherical shape (see Figure S2 in the Supporting Information). In contrast, the addition

$\dagger$ Condensed Matter and Interfaces, University Utrecht.

$¥$ Electron Microscopy Utrecht, University Utrecht.

\& University of Hamburg.

${ }^{\perp}$ Present address: Institute of Physical Chemistry and Electrochemistry,

Technical University of Dresden, Bergstrasse 66b, 01062 Dresden, Germany

6792 a J. AM. CHEM. SOC. 2006, 128, 6792-6793

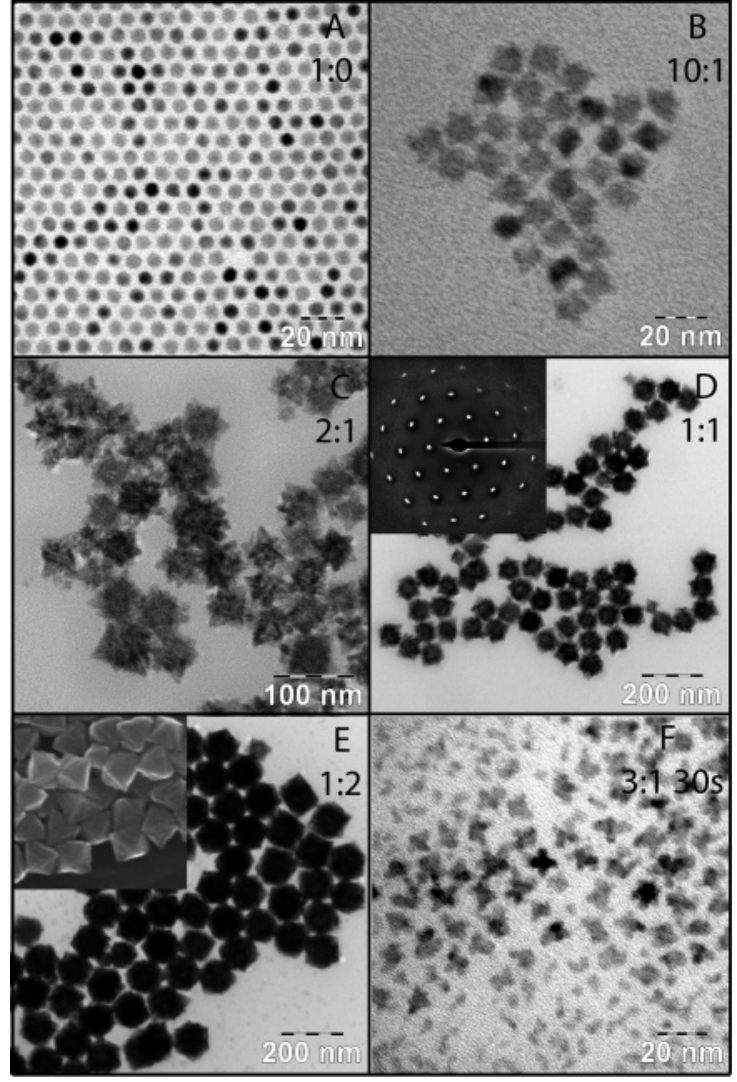

Figure 1. TEM images of $\mathrm{PbSe}$ crystal shapes grown at different concentrations of acetate. (A-E) PbSe NCs grown under identical reaction conditions with a $\mathrm{Pb}: \mathrm{Ac}$ ratio as indicated in the images. Insets are a diffraction pattern of a single octahedron (D) and a SEM image (E). (F) $\mathrm{PbSe} \mathrm{NCs}$ after $30 \mathrm{~s}$ of growth, in the initial stage of oriented attachment toward octahedrons.

of acetic acid to the injection mixture resulted in a dramatic change in crystal shape and size; see Figures 1B-E. From these images it is apparent that the addition of acetic acid leads to the starlike crystal shape; the amount of acetic acid has a strong effect on the size $(10-120 \mathrm{~nm})$ of these crystals. The "diameter" of the NCs is shown as a function of the $\mathrm{Pb}: \mathrm{Ac}$ ratio in Table $\mathrm{S} 1$.

The hexagons in the TEM images of the largest crystals (Figure 1E) are actually 2D projections of octahedrons, as can be clearly seen in a SEM image of the same NCs (inset in Figure 1E). The transition from the small spherical particles to the large octahedral particles involves an intermediate shape. The growth in the $\langle 100\rangle$ direction is faster, resulting in extensions in this direction. This leads to star-shaped particles that show up as stars of David in the TEM images (Figures 1B,C and S3A). For very large crystals the extensions in the $\langle 100\rangle$ direction become so large that all the $\{100\}$ 
facets have disappeared and perfect octahedrons result (Figures $1 \mathrm{E}$ and S3B-E). The formation of octahedrons by increasing the $\langle 100\rangle /$ $\langle 111\rangle$ growth ratio has previously been observed for $\mathrm{PbS} \mathrm{NCs}^{8}$

$\mathrm{Lu}$ et al. proposed that these octahedral crystals are formed by the aggregation of smaller PbSe clusters. ${ }^{5}$ Our observations confirm this mechanism. Figure $1 \mathrm{~F}$ shows a TEM image of PbSe crystals that were removed from the reaction mixture $30 \mathrm{~s}$ after the injection. A mixture of small crystallites of $\sim 4 \mathrm{~nm}$ in diameter and aggregates of 2-6 of those crystallites is visible in the initial stage of growth toward octahedrons. The final stars or octahedrons are single crystalline, as is illustrated by the discrete spots in the diffraction pattern of a single octahedron (inset in Figure 1D, Figure S4). These observations strongly suggest that those smaller crystallites selfassemble into the star-shaped PbSe crystals. This is essentially the same mechanism of oriented attachment that was proposed by Cho et $\mathrm{al}^{4}$ for the formation of $\mathrm{PbSe}$ nanowires and nanorings. The formation of octahedrons implies that the oriented attachment of smaller PbSe crystals is fastest in the $\langle 100\rangle$ direction, ultimately resulting in the elimination of all $\{100\}$ facets. This is in accordance with observations of Cho et al. ${ }^{4}$ In the formation of many different crystal shapes they observed only oriented attachment along the $\langle 100\rangle$ axis. As a possible explanation for this, they showed that the dipole moment of quasi-spherical particles is strongest along the $\langle 100\rangle$ axis and that this dipole orientation is the most frequent. ${ }^{4}$ The formation of octahedrons enhances this effect: the dipole increases with the area of the polar $\{111\}$ surface.

The addition of acetic acid to the reaction mixture leads to a (partial) replacement of oleate at the $\mathrm{Pb}$ sites on the $\mathrm{NC}$ surface. Because acetate is so much smaller than oleate, this strongly reduces steric hindrance between different crystals, which in turn drastically increases the rate of oriented attachment. This explains why an increasing concentration of acetate leads to larger NCs. The final size and shape of the crystals do not depend strongly on the growth temperature between 110 and $230{ }^{\circ} \mathrm{C}$. The acetate concentration is by far the most important parameter for this.

Lu et al. make no mention of the reason their synthesis results in star-shaped crystals. They prepare their $\mathrm{Pb}$-oleate precursors by heating to $150{ }^{\circ} \mathrm{C}$ for 30 min under an argon stream. ${ }^{5}$ We have used this exact drying procedure and found that it results in a mixture of quasi-spherical and octahedral NCs (see Figure S5). In contrast, Wehrenberg et al. and the Murray group report that they dry their precursors at $>85{ }^{\circ} \mathrm{C}$ under vacuum for at least $1 \mathrm{~h}^{3,9,10}$ when they prepare quasi-spherical PbSe NCs. Interestingly the Murray group reports the formation of octahedral-shaped $\mathrm{PbSe} \mathrm{NCs}$ when they do not dry under vacuum. This synthesis was performed in the presence of primary amines, and they speculate that the octahedral shape is caused by blocking of the $\{111\}$ facets by these amines. ${ }^{4}$ We have repeated this experiment, but with a reaction mixture that was carefully dried under vacuum, and we have observed only quasi-spherical NCs (see Figure S6). It is important to note that adding $4.8 \mathrm{mmol}$ of hexadecylamine (HDA) does not result in an apparent change in NC size or shape, whereas the addition of $0.17 \mathrm{mmol}$ of acetic acid has a marked effect on both (see Figure 1B). On the basis of the observations reported here we propose that heating the precursor mixture to $150{ }^{\circ} \mathrm{C}$ for $30 \mathrm{~min}$ under an argon stream leads to incomplete drying and the inherent presence of acetate at the surface of the synthesized NCs. We propose that this acetate is responsible for the high degree of

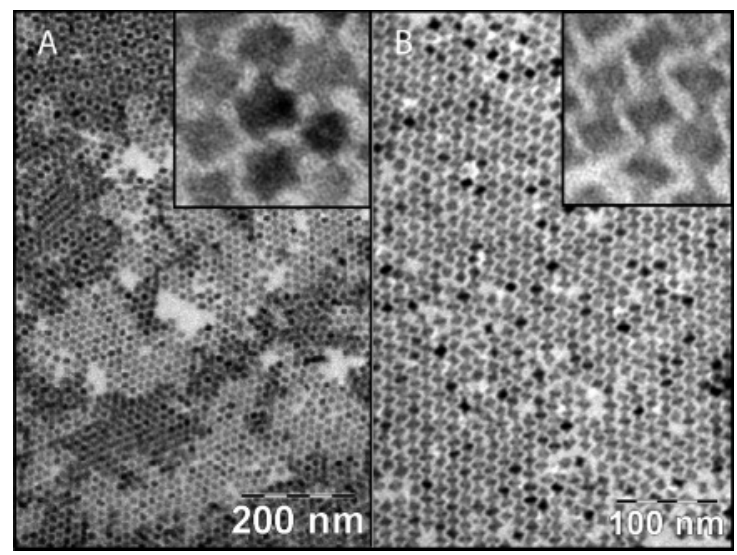

Figure 2. Self-assembled layers of star-shaped $\mathrm{PbSe}$ nanocrystals, viewed along the $\langle 111\rangle$ axis (A) and the $\langle 110\rangle$ axis (B).

oriented attachment and many of the resulting crystal shapes reported in the literature..$^{4,5,11}$

In the presence of small amounts of acetate $(\mathrm{Pb}: \mathrm{Ac} \approx 5: 1)$ the star-shaped NCs can be made very monodisperse. The narrow size distribution results in a striking long-range order in the 2-D packing of star-shaped NCs (see Figure 2) and crystal alignment at the $\{100\}$ faces (see also Figure S7). The reason for this alignment may again be the dipole moment along the $\langle 100\rangle$ axis.

The formation of self-assembled structures of anisotropic NCs with crystal alignment offers possibilities for the engineering of functional NC solids. The expected anisotropy in the quantum mechanical coupling may lead to new classes of electronic materials. The optical and electrical properties of such a self-assembled structure may strongly depend on the direction that is probed.

In conclusion, we have shown that acetate, naturally present in insufficiently dried reaction mixtures, is a key factor in the growth mechanism and the final PbSe $\mathrm{NC}$ shape and size.

Acknowledgment. Financial support from the European Union network "FULLSPECTRUM" (SES6-CT-2003-502620) is gratefully acknowledged.

Supporting Information Available: Experimental details and TEM images of different syntheses. This material is available free of charge via the Internet at http://pubs.acs.org.

\section{References}

(1) Wise, F. W. Acc. Chem. Res. 2000, 33, 773.

(2) Murray, C. B.; Sun, S. H.; Gaschler, W.; Doyle, H.; Betley, T. A.; Kagan, C. R. IBM J. Res. Dev. 2001, 45, 47

(3) Wehrenberg, B. L.; Wang, C. J.; Guyot-Sionnest, P. J. Phys. Chem. B 2002, 106, 10634 .

(4) Cho, K. S.; Talapin, D. V.; Gaschler, W.; Murray, C. B. J. Am. Chem. Soc. 2005, 127, 7140.

(5) Lu, W. G.; Fang, J. Y.; Ding, Y.; Wang, Z. L. J. Phys. Chem. B 2005, 109, 19219

(6) Lifshitz, E.; Bashouti, M.; Kloper, V.; Kigel, A.; Eisen, M. S.; Berger, S. Nano Lett. 2003, 3, 857.

(7) Pietryga, J. M.; Schaller, R. D.; Werder, D.; Stewart, M. H.; Klimov, V I.; Hollingsworth, J. A. J. Am. Chem. Soc. 2004, 126, 11752.

(8) Lee, S. M.; Jun, Y. W.; Cho, S. N.; Cheon, J. J. Am. Chem. Soc. 2002 124, 11244.

(9) Shevchenko, E. V.; Talapin, D. V.; O’Brien, S.; Murray, C. B. J. Am Chem. Soc. 2005, 127, 8741 .

(10) Talapin, D. V.; Murray, C. B. Science 2005, 310, 86

(11) Lu, W. G.; Gao, P. X.; Bin Jian, W.; Wang, Z. L.; Fang, J. Y. J. Am Chem. Soc. 2004, 126, 14816

JA061644V 